\title{
Determination of Rounded Edge Diffraction Loss for a Plateau Using Hacking Method
}

\author{
Victor Akpaiya Udom, Kalu Constance, Asuquo Ifiok Okon \\ Department of Electrical/Electronic and Computer Engineering, University of Uyo, Uyo, Nigeria
}

Email address:

constance.kalu@yahoo.com (K. Constance)

\section{To cite this article:}

Victor Akpaiya Udom, Kalu Constance, Asuquo Ifiok Okon. Determination of Rounded Edge Diffraction Loss for a Plateau Using Hacking Method. American Journal of Software Engineering and Applications. Vol. 6, No. 2, 2017, pp. 35-39. doi: 10.11648/j.ajsea.20170602.14

Received: January 3, 2017; Accepted: January 18, 2017; Published: June 12, 2017

\begin{abstract}
In this paper, Hacking rounded edge diffraction loss method is used to determine the diffraction loss over a plateau in the path of microwave signal in the GSM frequency band, $800 \mathrm{MHz}$ to $2100 \mathrm{MHz}$. The computation is based on the path profile with path length of $4996.243 \mathrm{~m}$ and a plateau in the signal path. The plateau has maximum elevation of $268.9 \mathrm{~m}$ and it occurred at a distance of $3557.8 \mathrm{~m}$ from the transmitter. The line of sight clearance height is $45.747499 \mathrm{~m}$ and occultation distance is $1538.759 \mathrm{~m}$. At $800 \mathrm{MHz}$, the diffraction loss is $55.25 \mathrm{~dB}$ whereas at $2100 \mathrm{MHz}$ the diffraction loss is $71.713 \mathrm{~dB}$. The result is useful for GSM network planning.
\end{abstract}

Keywords: Rounded Edge Diffraction, Diffraction Loss, Elevation Profile, Diffraction Parameter, Knife Edge Diffraction, Hacking Rounded Edge Diffraction Method

\section{Introduction}

A plateau is an area of fairly level high ground. When an isolated plateau is located in the path of microwave signal it constitutes an obstruction. As an obstruction, the plateau will cause diffraction loss [1-5]. Like other obstructions such as hill and building, the plateau can be approximated as knife edge obstruction. However, approximation will under-estimate the diffraction loss that can be caused by the plateau $[6,7]$. In that case, a rounded edge obstruction approximation is preferable [8-14].

In this paper, determination of rounded edge diffraction loss for a plateau using Hacking method [15-19] is presented for $800 \mathrm{MHz}$ to $2100 \mathrm{MHz}$ GSM (Global System for Mobile communication) frequency band. The study utilizes the path profile of a microwave link with a plateau in the signal path to construct the rounded edge geometry which is then used to determine the rounded edge diffraction loss expected from the plateau.

\section{Theoretical Background}

\subsection{The Rounded Plateau Obstruction Geometry and Parameters}

Figure 1 shows the elevation profile of a plateau obstruction between the transmitter $(\mathrm{T})$ and the receiver $(\mathrm{R})$.

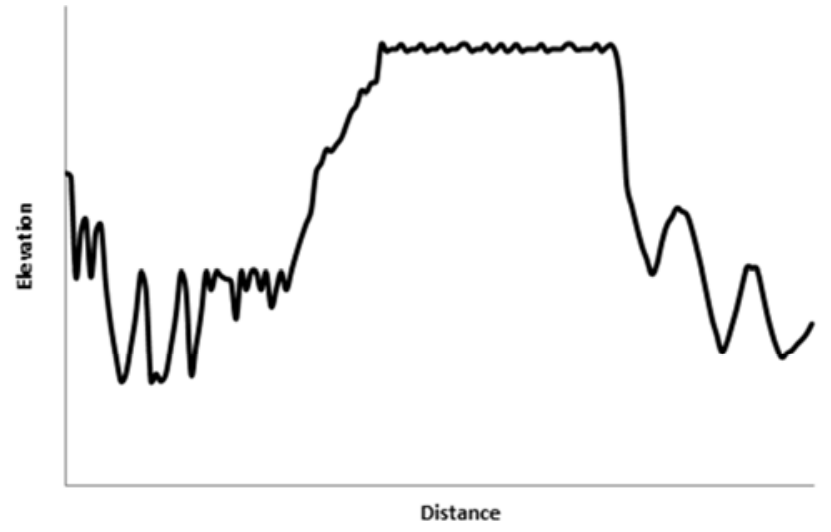

Figure 1. The Elevation Profile Of A Plateau As The Obstruction In The Signal Path.

In order to determine the diffraction loss that the plateau will present using the rounded edge approach, a tangent (denoted in this paper as tangent line $\mathrm{t}$ ) must be drawn from the transmitter to the elevation profile at the vicinity of the plateau vertex, as shown in figure 2. Similar tangent line (denoted in this paper as tangent line r) is also drawn from the receiver to the elevation profile at the vicinity of the plateau vertex. Then, a circle is fitted to the plateau at the vicinity of the plateau vertex in such a 
way that the circle is tangential to the two tangential lines to the elevation profile. In this paper, the tangent line $t$, the tangent line $r$, their tangent points and their lengths denoted as S1 and $\mathrm{S} 2$ are determined by drawing the tangent line $\mathrm{t}$ and tangent line $r$ on the graphical plot of the path profile. Also, the length of the line of sight (LOS) denoted as S3 is measured out from the graph plot of the path profile.

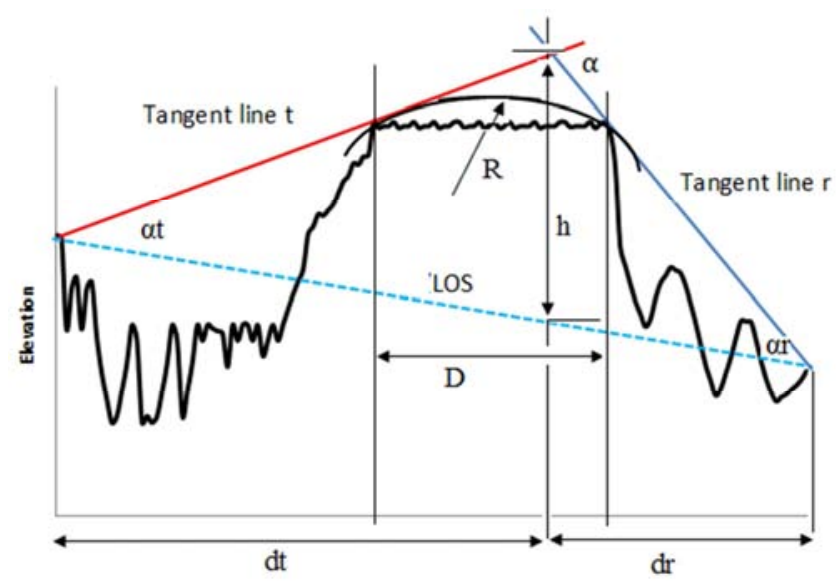

Figure 2. The Elevation Profile Of The Plateau Obstruction With Tangential Lines And Fitted Circle At The Vicinity Of The Plateau Vertex.

Figure 2 shows the essential parameters required for the rounded plateau top diffraction loss computation. The key dimensions include:

i. S1: the length of (tangent line $t$ ) the tangent from the transmitter to the intersection point of tangent line $t$ and tangent line $r$.

ii. S2: the length of (tangent line $r$ ) the tangent from the receiver to the intersection point of tangent line $t$ and tangent line $r$.

iii. S3: the length of the line of sight (LOS) which is the line from the transmitter to the receiver.

iv. $\beta$ : The LOS is inclined at angle $\beta$ to the horizontal. The angle which the LOS makes with the horizontal is denoted as $\beta$ where;

$$
\beta=\tan ^{-1}\left(\frac{\mathrm{H}_{\mathrm{elt}}-\mathrm{H}_{\mathrm{elr}}}{\mathrm{d}}\right)
$$

where

$\mathrm{H}_{\text {elt }}$ is the elevation of the transmitter and $\mathrm{H}_{\mathrm{elr}}$ is the elevation of the receiver. $\mathrm{H}_{\text {elt }}$ and $\mathrm{H}_{\text {elr }}$ are obtained from the path profile data.

v. d1: the horizontal distance from the transmitter to the intersection point of the two tangents

vi. d2: the horizontal distance from the receiver to the intersection point of the two tangents

vii. $d$ is the path length, that is the distance between the transmitter and the receiver, and $d$ is given as;

$$
\mathrm{d}=\mathrm{d}_{\mathbf{t}}+\mathrm{d}_{\mathbf{r}}
$$

$\mathrm{d}_{\mathrm{t}}$ and $\mathrm{d}_{\mathrm{r}}$ are measured from the graph plot and the intersection point of tangent line $t$ and tangent line $r$

viii. $\mathrm{D}$ : the occultation distance, which is the distance between the tangent point before and the tangent point after the intersection point of tangent line $t$ and tangent line $t . D$ is the distance between the tangent point of tangent line $\mathrm{t}$ with the path profile and the tangent point of tangent line $r$ with the path profile. In this paper, the tangent points are determined by drawing the tangent line $t$ and tangent line $r$ on the graphical plot of the path profile. Then, D is measured from the graph plot as the distance between the tangent points of tangent line $t$ and tangent line $r$.

ix. at: the angle between the LOS and (tangent line $t$ ) the tangent line drawn from the transmitter to the elevation profile.

x. $\quad$ r: the angle between the LOS and (tangent line $r$ ) the tangent line drawn from the receiver to the elevation profile.

The angles $\alpha \mathrm{t}$ and $\alpha \mathrm{r}$ are obtain by cosine rule as follows;

$$
\begin{gathered}
\operatorname{Cos}(\alpha \mathrm{t})=\frac{(\mathrm{S} 1)^{2}+(\mathrm{S} 3)^{2}-(\mathrm{S} 2)^{2}}{2(\mathrm{~S} 1)(\mathrm{S} 3)} \\
\alpha \mathrm{t}=\mathrm{Cos}^{-1}\left(\frac{(\mathrm{S} 1)^{2}+(\mathrm{S} 3)^{2}-(\mathrm{S} 2)^{2}}{2(\mathrm{~S} 1)(\mathrm{S} 3)}\right)
\end{gathered}
$$

Similarly,

$$
\alpha \mathrm{r}=\operatorname{Cos}^{-1}\left(\frac{(\mathrm{S} 1) 2^{2}+(\mathrm{S} 3)^{2}-(\mathrm{S} 1)^{2}}{2(S 2)(S 3)}\right)
$$

The angle $\alpha$ is given as;

$$
\alpha=\alpha \mathrm{t}+\alpha \mathrm{r}
$$

xi. $h$ is the height of the intersection point of tangent line $\mathrm{t}$ and tangent line $\mathrm{r}$ above the LOS. $h$ is the height of the intersection point of tangent line $t$ and tangent line $\mathrm{r}$ above the LOS. $\mathrm{h}$ is given as;

$$
h=\frac{S 1[\operatorname{Sin}(\alpha t)]}{\operatorname{Sin}(90-\beta)}
$$

xii. $\mathrm{v}$ is he diffraction parameter which is given as;

$$
\mathrm{v}=h \sqrt{\frac{2(d 1+d 2)}{\lambda(\mathrm{d} 1)(\mathrm{d} 2)}}
$$

xiii. $\mathrm{R}$ is the radius of the circle fitted to the plateau in the vicinity of the plateau vertex. The radius of the circle fitted in the vicinity of the hill vertex. The circle fitted in the vicinity of the hill vertex is tangential to the tangent line $t$ and tangent line $r$. The approximate value of $\mathrm{R}$ is estimated from the path profile using the formula [20, 21];

$$
R=\frac{2(\mathrm{D})(\mathrm{d} 1)(\mathrm{d} 2)}{(\alpha)\left[\left(\mathrm{d} 1^{2}\right)+\left(\mathrm{d} 2^{2}\right)\right]}
$$

\subsection{Hacking Method for Computing Diffraction Loss over Rounded Edge}

According to Hacking method for diffraction loss on rounded edge, the total diffraction loss, $\mathrm{A}_{d B}(v)$ is given as;

$$
\mathrm{A}_{d B}(v)=\mathrm{A}_{k e}+A_{e x}
$$


Where

$\mathrm{A}_{k e}$ is the knife edge diffraction loss

$\mathrm{v}$ is the diffraction parameter as given in Eq 8 .

$A_{e x}$ is the excess diffraction loss which is added to the

knife edge diffraction loss to account for the rounded hilltop.
The knife edge diffraction loss, $\mathrm{A}_{k e}$ can be determined by Lee's piecewise knife edge diffraction loss approximation model expressed in respect of diffraction parameter, $\mathrm{v}$ as follows $[22,23]$ :

$$
\mathrm{A}_{k e}(d B)=\left\{\begin{array}{c}
0 \text { for } \mathrm{v}<-1 \\
20 \log (0.5-0.62 \mathrm{v}) \text { for }-1 \leq \mathrm{v} \leq 0 \\
20 \log (0.5 \exp (-0.95 \mathrm{v})) \text { for } 0 \leq \mathrm{v} \leq 1 \\
20 \log \left(0.4-\sqrt{0.1184-(0.38-0.1 \mathrm{v})^{2}}\right) \text { for } 1 \leq \mathrm{v} \leq 2.4 \\
20 \log \left(\frac{0.225}{v}\right) \text { for } \mathrm{v}>2.4
\end{array}\right\}
$$

where

- $\mathrm{v}$ is the diffraction parameter as given in Eq 8 .

- $\mathrm{h}$ is the line of sight (LOS) clearance height, $\mathrm{h}$ is in meters;

- $d_{t}$ is the distance from the transmitter and $d_{r}$ is the distance from the receiver. $d_{t}$ and $d_{r}$ are is $\mathrm{km}$.

The excess diffraction loss according to Hacking is given as $[15,16]$ :

$$
\mathrm{A}_{\mathrm{ex}}=11.7(\alpha)\left(\frac{(\pi) \mathrm{R}}{\lambda}\right)^{1 / 3}
$$

Where

- $\alpha$ is the exterior angle (in radian) between the tangent line drawn from the transmitter (referred to as tangent line $t$ ) and the tangent lines drawn from the receiver (referred to as tangent line r). $\alpha$ is given from $\mathrm{Eq} 6, \mathrm{Eq} 3$ and $\mathrm{Eq} 5$.

- $\mathrm{R}$ is the radius of the circle fitted in the vicinity of the obstruction vertex. $\mathrm{R}$ is given in Eq 9.

- $K$ is the signal wavelength which is given as;

$$
\Lambda=\frac{c}{f}
$$

$\mathrm{f}$ is the frequency in $\mathrm{Hz}$ and $\mathrm{c}$ is the speed of light which is $3 \times 10^{8} \mathrm{~m} / \mathrm{s}$.

\section{Results and Discussions}

The study if conducted for the L-band microwave frequency which ranges from $1 \mathrm{GHz}$ to $2 \mathrm{GHz}$. Specifically, the $1 \mathrm{GHz}$ and $1.9 \mathrm{GHz}$ frequencies are considered in this paper. The elevation profile data used for the study is given in Table 1 . From Table 1 the elevation maximum elevation is $268.9 \mathrm{~m}$ and

\begin{tabular}{|c|c|c|c|c|c|c|c|c|c|}
\hline $\begin{array}{l}\text { Distance from the } \\
\text { transmitter (m) }\end{array}$ & $\begin{array}{l}\text { Elevation } \\
(\mathbf{m})\end{array}$ & $\begin{array}{l}\text { Distance from the } \\
\text { transmitter }(\mathbf{m})\end{array}$ & $\begin{array}{l}\text { Elevation } \\
(\mathrm{m})\end{array}$ & $\begin{array}{l}\text { Distance from the } \\
\text { transmitter }(m)\end{array}$ & $\begin{array}{l}\text { Elevation } \\
(\mathbf{m})\end{array}$ & $\begin{array}{l}\text { Distance from the } \\
\text { transmitter }(\mathrm{m})\end{array}$ & $\begin{array}{l}\text { Elevation } \\
(\mathbf{m})\end{array}$ & $\begin{array}{l}\text { Distance from the } \\
\text { transmitter }(\mathrm{m})\end{array}$ & $\begin{array}{l}\text { Elevation } \\
\text { (m) }\end{array}$ \\
\hline 0.0 & 250.3 & 1015.5 & 235.7 & 2019.1 & 262.0 & 3022.6 & 268.9 & 4026.2 & 242.4 \\
\hline 45.5 & 249.7 & 1049.0 & 235.2 & 2052.5 & 263.3 & 3056.1 & 267.9 & 4059.6 & 243.7 \\
\hline 78.9 & 235.0 & 1082.4 & 234.7 & 2086.0 & 263.6 & 3089.5 & 268.2 & 4093.1 & 245.0 \\
\hline 112.4 & 241.5 & 1115.9 & 234.4 & 2119.4 & 268.9 & 3123.0 & 268.2 & 4126.5 & 244.6 \\
\hline 145.8 & 243.3 & 1149.3 & 228.9 & 2152.9 & 267.9 & 3156.4 & 268.3 & 4160.0 & 244.1 \\
\hline 179.3 & 235.0 & 1182.8 & 235.7 & 2186.3 & 268.2 & 3189.9 & 268.9 & 4193.4 & 242.0 \\
\hline 246.2 & 242.6 & 1249.7 & 235.7 & 2253.2 & 268.9 & 3256.8 & 268.2 & 4260.3 & 236.0 \\
\hline 279.6 & 233.8 & 1283.1 & 235.7 & 2286.7 & 267.9 & 3290.2 & 268.2 & 4293.8 & 232.4 \\
\hline 313.1 & 227.9 & 1316.6 & 233.2 & 2320.1 & 268.2 & 3323.7 & 268.3 & 4327.2 & 229.0 \\
\hline 346.5 & 223.4 & 1350.1 & 235.7 & 2353.6 & 268.3 & 3357.1 & 268.9 & 4360.7 & 226.5 \\
\hline 380.0 & 219.6 & 1383.5 & 230.6 & 2387.0 & 268.9 & 3390.6 & 268.9 & 4394.1 & 224.0 \\
\hline 413.4 & 220.7 & 1417.0 & 233.2 & 2420.5 & 267.9 & 3424.0 & 268.1 & 4427.6 & 225.4 \\
\hline 446.9 & 224.9 & 1450.4 & 235.7 & 2453.9 & 268.2 & 3457.5 & 268.2 & 4461.0 & 227.9 \\
\hline 480.3 & 229.5 & 1483.9 & 233.2 & 2487.4 & 268.3 & 3490.9 & 268.2 & 4494.5 & 230.5 \\
\hline 547.2 & 233.2 & 1550.8 & 238.2 & 2554.3 & 267.9 & 3557.8 & 268.9 & 4561.4 & 236.4 \\
\hline 580.7 & 219.6 & 1584.2 & 240.6 & 2587.7 & 268.2 & 3591.3 & 267.9 & 4594.8 & 236.3 \\
\hline 614.1 & 220.7 & 1617.7 & 242.8 & 2621.2 & 268.3 & 3624.7 & 268.5 & 4628.3 & 236.2 \\
\hline 647.6 & 219.6 & 1651.1 & 244.7 & 2654.7 & 268.9 & 3658.2 & 268.8 & 4661.7 & 233.2 \\
\hline 681.0 & 220.7 & 1684.6 & 250.5 & 2688.1 & 268.9 & 3691.6 & 267.2 & 4695.2 & 229.7 \\
\hline 714.5 & 224.9 & 1718.0 & 251.7 & 2721.6 & 267.9 & 3725.1 & 261.8 & 4728.6 & 226.8 \\
\hline 747.9 & 229.5 & 1751.5 & 253.7 & 2755.0 & 268.2 & 3758.5 & 248.5 & 4762.1 & 224.6 \\
\hline 781.4 & 235.7 & 1784.9 & 253.5 & 2788.5 & 268.3 & 3792.0 & 245.3 & 4795.5 & 223.1 \\
\hline 814.8 & 233.2 & 1818.4 & 254.4 & 2821.9 & 268.9 & 3825.4 & 242.3 & 4829.0 & 223.8 \\
\hline 848.3 & 220.7 & 1851.8 & 255.4 & 2855.4 & 267.9 & 3858.9 & 239.5 & 4862.4 & 224.3 \\
\hline 881.7 & 224.9 & 1885.3 & 257.3 & 2888.8 & 268.3 & 3892.4 & 237.5 & 4895.9 & 225.1 \\
\hline 915.2 & 229.5 & 1918.7 & 259.2 & 2922.3 & 268.9 & 3925.8 & 235.4 & 4929.3 & 225.8 \\
\hline 948.6 & 235.7 & 1952.2 & 260.1 & 2955.7 & 267.9 & 3959.3 & 236.7 & 4962.8 & 226.8 \\
\hline 982.1 & 233.2 & 1985.6 & 262.2 & 2989.2 & 268.3 & 3992.7 & 239.9 & 4996.2 & 228.2 \\
\hline
\end{tabular}
it occurred at a distance of $3557.8 \mathrm{~m}$ from the transmitter.

Table 1. The Elevation Profile For The Plateau. 
Table 2. Rounded Edge Parameters For The Plateau Obstruction

\begin{tabular}{|c|c|c|}
\hline $\mathbf{f}(\mathbf{G H z})$ & Frequency & 1000 \\
\hline$\lambda(\mathrm{m})$ & Wavelength & 0.3 \\
\hline $\mathrm{S} 1(\mathrm{~m})$ & The length of the tangent from the transmitter to the intersection point of the two tangent & 3283.0914 \\
\hline $\mathrm{S} 2(\mathrm{~m})$ & the length of the tangent from the receiver to the intersection point of the two tangents & 1714.1298 \\
\hline $\mathrm{S} 3(\mathrm{~m})$ & the length of the tangent from the receiver from the transmitter & 4996.2918 \\
\hline $\mathrm{dt}(\mathrm{m})$ & the distance from the transmitter to the intersection point of the two tangents, that is point & 3282.9428 \\
\hline $\mathrm{dr}(\mathrm{m})$ & the distance from the receiver to the intersection point of the two tangents & 1713.3002 \\
\hline $\mathrm{d}(\mathrm{m})$ & the distance from the transmitter to the receiver & 4996.243 \\
\hline$\alpha \mathrm{t}$ (radian) & The angle the tangent line from the transmitter makes with the LOS & 0.0266914 \\
\hline$\alpha \mathrm{r}$ (radian) & The angle the tangent line from the receiver makes with the LOS & 0.0139346 \\
\hline$\alpha($ radian $)$ & Sum of angles $\alpha \mathrm{t}$ and $\alpha \mathrm{r}$ & 0.040626 \\
\hline$\beta$ (radian) & The angle the LOS makes with the horizontal & 0.0044209 \\
\hline $\mathrm{h}(\mathrm{m})$ & The LOS clearance height & 45.747499 \\
\hline $\mathrm{D}(\mathrm{m})$ & The occultation distance & 1538.759 \\
\hline $\mathrm{R}(\mathrm{m})=$ & The radius of the circle fitted in the vicinity of the hill vertex & 31071.175 \\
\hline
\end{tabular}

Table 2 shows the key rounded edge parameters obtained for the plateau obstruction. From Table 2, the path length (d) is $4996.243 \mathrm{~m}$. Also, the tangent from the transmitter and the tangent from the receiver intersected at a distance of $3282.9428 \mathrm{~m}$ from the transmitter and a distance of $1713.3002 \mathrm{~m}$ from the receiver. The line of sight makes an angle of 0.0044209 radians with the horizontal. The LOS clearance height is $45.747499 \mathrm{~m}$. The occultation distance is $1538.759 \mathrm{~m}$.

Table 3 and figure 3 show the diffraction parameter and the diffraction loss for the rounded edge plateau as computed by the Hacking method. The diffraction parameter and also the diffraction loss increases with frequency. At $800 \mathrm{MHz}$, the plateau will cause diffraction loss of $55.25 \mathrm{~dB}$ whereas at 2100 $\mathrm{MHz}$ the plateau will cause diffraction loss of $71.713 \mathrm{~dB}$.

Table 3. The Diffraction Parameter and The Diffraction Loss For The Rounded Edge Plateau Computed by The Hacking Method.

\begin{tabular}{lll}
\hline Frequency (MHz) & Diffraction Parameter, V & Diffraction Loss (dB) \\
\hline 800 & 3.148764 & 55.250 \\
900 & 3.339769 & 57.051 \\
1800 & 4.723146 & 68.804 \\
1900 & 4.852571 & 69.811 \\
2100 & 5.101581 & 71.713 \\
\hline
\end{tabular}

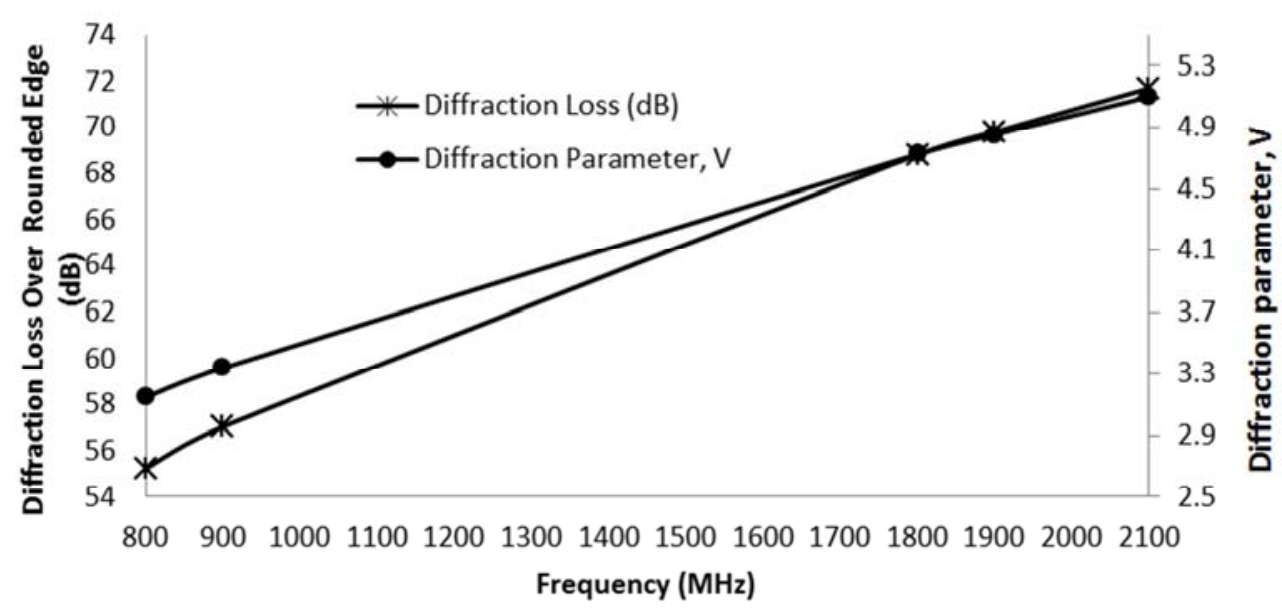

Figure 3. The Diffraction Parameter and The Diffraction Loss For The Rounded Edge Plateau Computed by The Hacking Method.

\section{Conclusions}

Hacking rounded edge diffraction loss method is presented. The method is used to determine the diffraction loss over a plateau in the path of microwave signal in the GSM frequency band, $800 \mathrm{MHz}$ to $2100 \mathrm{MHz}$. The computation is based on the path profile of a plateau. The results show how the diffraction parameter and the diffraction loss vary with frequencies within the given $800 \mathrm{MHz}$ to $2100 \mathrm{MHz}$ frequency band.

\section{References}

[1] Rao, P. S. (2013) Performance Analysis of Diffraction Gain (Gd) Due to presence of Knife-Edge as Compared to Free Space E-Field and Identifying the Position of Obstacle in a Fresnel Zone. International Journal of Scientific and Research Publications, Volume 3, Issue 1, January 2013.

[2] He, R., Molisch, A. F., Tufvesson, F., Zhong, Z., Ai, B., \& Zhang, T. (2014). Vehicle-to-vehicle propagation models with large vehicle obstructions. IEEE Transactions on Intelligent Transportation Systems, 15 (5), 2237-2248. 
[3] Akkaşl1, C. (2009). Methods for Path loss Prediction. Reports from MSI, School of Mathematics and Systems Engineering, Report, 9067.

[4] Fickenscher, M. R. T. (2014, March). Diffraction loss and phase modulation of terrestrial radio-link by wind turbine. In Antenna Technology:" Small Antennas, Novel EM Structures and Materials, and Applications" (iWAT), 2014 International Workshop on (pp. 382-384). IEEE.

[5] Klostius, R., Wieser, A., \& Brunner, F. K. (2006, May). Treatment of diffraction effects caused by mountain ridges. In Proceedings of 3rd IAG/12th FIG Symposium, Baden.

[6] Durgin, G. D. (2009). The practical behavior of various edge-diffraction formulas. IEEE Antennas and Propagation Magazine, 51 (3), 24-35.

[7] Durgin, G. D. (2008, July). Practical geometrical behavior of knife-edge diffraction. In 2008 IEEE Antennas and Propagation Society International Symposium (pp. 1-4). IEEE.

[8] Gnani, F., Lo, K. H., Zare-Behtash, H., \& Kontis, K. (2014). Experimental investigation on shock wave diffraction over sharp and curved splitters. Acta Astronautica, 99, 143-152.

[9] Kizer, G. (2013). Digital microwave communication: engineering point-to-point microwave systems. John Wiley \& Sons.

[10] Vogler, L. E. (1985). Radio wave diffraction by a rounded obstacle. Radio science, 20 (3), 582-590.

[11] Gnani, F., Lo, K. H., Zare-Behtash, H., \& Kontis, K. (2015). Shock Wave Diffraction Phenomena around Slotted Splitters. Aerospace, 2 (1), 1-16.

[12] Emerson, D., \& Lewis, M. (2004). Propagation Models. Lewis \& Emerson (2004), 48

[13] Rathsam, J., \& Wang, L. M. (2007). Scattered Responses From Suspended Reflector Panels With Rounded Edges.
[14] Mack, C. A. (2000). Corner rounding and round contacts. Microlithography World, summer.

[15] Parsons, J. D., \& Parsons, P. J. D. (2000). The mobile radio propagation channel. Second edition. John Wiley \& Sons.

[16] Hacking, K. U. H. F. (1968). Propagation over rounded hills. BBC Research Department. Research Report No. RA-21, 30.

[17] Hill, G. (2007). The Cable and Telecommunications Professionals' Reference: PSTN, IP and cellular networks, and mathematical techniques (Vol. 1). Taylor \& Francis.

[18] McArthur, R. J., \& Bebbington, D. H. O. (1991, April). Diffraction over simple terrain obstacles by the method of parabolic equations. In Antennas and Propagation, 1991. ICAP 91., Seventh International Conference on (IEE) (pp. 824-827). IET.

[19] Hacking K., (1970) "U. H. F. propagation over rounded hills", Proc. IEE, vol. 117, PP. 499-511, 1970.

[20] Seybold, J. S. (2005). Introduction to RF propagation. John Wiley \& Sons.

[21] Barué, G. (2008). Microwave engineering: land \& space radiocommunications (Vol. 9). John Wiley \& Sons.

[22] Jude, O. O., Jimoh, A. J., \& Eunice, A. B. (2016). Software for Fresnel-Kirchoff Single Knife-Edge Diffraction Loss Model. Mathematical and Software Engineering, 2 (2), 76-84.

[23] Rodriguez, I., Nguyen, H. C., Sørensen, T. B., Zhao, Z., Guan, H., \& Mogensen, P. (2016, October). A novel geometrical height gain model for line-of-sight urban micro cells below 6 GHz. In Wireless Communication Systems (ISWCS), 2016 International Symposium on (pp. 393-398). IEEE. 